\title{
Pretreatment assessment and perioperative nutritional interventions for patients with bladder cancer undergoing radical cystectomy
}

\author{
Makito Miyake, Kiyohide Fujimoto \\ Department of Urology, Nara Medical University, Kashihara-shi, Nara 634-8522, Japan \\ Correspondence to: Makito Miyake. Department of Urology, Nara Medical University, 840 Shijo-cho, Kashihara-shi, Nara 634-8522, Japan. \\ Email: makitomiyake@yahoo.co.jp. \\ Provenance: This is a Guest Editorial commissioned by Section Editor Xiao Li (Department of Urology, Jiangsu Cancer Hospital \& Jiangsu Institute \\ of Cancer Research \& Nanjing Medical University Affiliated Cancer Hospital, Nanjing, China). \\ Comment on: Hamilton-Reeves JM, Stanley A, Bechtel MD, et al. Perioperative Immunonutrition Modulates Inflammatory Response after Radical \\ Cystectomy: Results of a Pilot Randomized Controlled Clinical Trial. J Urol 2018;200:292-301.
}

Submitted Jul 02, 2018. Accepted for publication Jul 03, 2018.

doi: $10.21037 /$ tau.2018.07.02

View this article at: http://dx.doi.org/10.21037/tau.2018.07.02

In a recent issue of the fournal of Urology, Hamilton-Reeves et al. (1) reported interesting results of a prospective, randomized study that sought to compare the effects of a short-term specialized immunonutrition (SIM) with those of a standard oral nutrition supplement (ONS) on the acute inflammatory response and arginine status in patients with bladder cancer undergoing radical cystectomy (RC). RC involves several invasive procedures and is associated with high rates of complications and morbidity, partly due to extensive lymph node dissection and urinary diversions using intestinal segments, for example, orthotopic neobladders and ileal conduits. Previous studies demonstrated that preoperative nutritional status, inflammatory status, host immunity, and muscle volume are strongly linked with not only complications, but oncological outcomes as well (2-6). Our group has emphasized the association between poor outcomes and post-RC changes in psoas muscle mass and nutritional index (4). This evidence suggested that prevention of the RC-induced deterioration of skeletal muscle mass and nutrition status could improve the clinical outcome including the complications, healthrelated quality of life, and cancer prognosis. To date, multiple measurements are available to evaluate the immunonutritional status and physical performance in patients with malignant diseases (Figure 1). Physicians should cautiously evaluate the patient condition before, during, and after the treatment.
Although several studies have established the nutritional and immunological benefits of immune-enhancing nutritional supplements for patients undergoing upper and lower gastrointestinal surgery $(7,8)$, there is a significant lack of guidelines and evidence for immunonutrition usage in the urologic oncology field. Munbauhal et al. provided an overview of malnourishment in patients with bladder cancer and the clinical role and benefit of immunonutrition at the time of RC (9). In this review, the prevalence of severe malnutrition was found to be $16-33 \%$, which was detected as a strong predictor of 90-day mortality. A clinical trial by Roth et al. (10) found that TPN IM (Nutriflex) conferred a significant impact on protein levels (prealbumin and total protein) 7 days after RC compared with those in the control arm (Ringer's lactate), and a lower rate of postoperative ileus $(16.2 \%$ vs. $9.6 \%)$. In contrast to the encouraging result, two studies by Maffezzini et al. failed to prove the benefit of the Enteral IM (IMPACT) provision $(11,12)$. More robust evidence is needed to establish the guidelinebased recommendation of immune-enhancing nutritional supplements for patients undergoing RC.

The results of a well-designed randomized control trial by Hamilton-Reeves et al. have been published in the Fournal of Urology (1). Participants received 3 cartons per day of either Impact Advanced Recovery ${ }^{\circledR}$ (SIM), containing L-arginine, nucleotides, and fish oil, or Boost Plus ${ }^{\circledR}$ (ONS, a control arm) provided from Nestlé Health Science for 


\begin{tabular}{|c|c|}
\hline \multicolumn{2}{|c|}{ Muscle mass } \\
\hline$\checkmark$ Computed tomograpy (CT)
\end{tabular}

Figure 1 Measurements of immunonutritional status and physical performance in patients with malignant diseases.

5 days before and 5 days after RC. A unique aspect of this study is that the researchers investigated the perioperative changes in multiple parameters, including $\mathrm{T}$ helper 1 (Th1)-Th2 balance, interleukin (IL)-6, plasma amino acids, generated subjective global assessment, skeletal muscle, and patient-reported quality of life. In summary, perioperative administration of SIM can preferably modulate the inflammatory response after RC. Thus, results support the intervention of SIM to maintain Th1-Th2 balance and lower plasma IL-6, which could reduce postoperative complications and muscle wasting $(13,14)$. One of the limitations of this study is the lack of detailed information regarding postoperative and long-term complications and oncological outcomes.

Another concern is whether specialized immunonutrition supplements are beneficial for all patients undergoing RC. Although this intervention is simple and noninvasive, a selection of patients who are expected to benefit should be considered. Baseline characteristics, including nutritional index and physical condition (Figure 1) before RC, may be useful for patient selection criteria for appropriate perioperative interventions. Accumulating evidence supports perioperative immunonutrition supplements lead to improved postoperative outcomes in patients undergoing $\mathrm{RC}$; however, there is an absolute need for larger randomized control trials in this population.

\section{Acknowledgements}

None.

\section{Footnote}

Conflicts of Interest: The authors have no conflicts of interest to declare.

\section{References}

1. Hamilton-Reeves JM, Stanley A, Bechtel MD, et al. Perioperative Immunonutrition Modulates Inflammatory 
Response after Radical Cystectomy: Results of a Pilot Randomized Controlled Clinical Trial. J Urol 2018;200:292-301.

2. Miyake M, Morizawa Y, Hori S, et al. Integrative Assessment of Pretreatment Inflammation-, Nutrition-, and Muscle-Based Prognostic Markers in Patients with Muscle-Invasive Bladder Cancer Undergoing Radical Cystectomy. Oncology 2017;93:259-69.

3. Morizawa Y, Miyake M, Shimada K, et al. Neutrophilto-lymphocyte ratio as a detection marker of tumor recurrence in patients with muscle-invasive bladder cancer after radical cystectomy. Urol Oncol 2016;34:e11-7.

4. Miyake M, Morizawa Y, Hori S, et al. Clinical impact of postoperative loss in psoas major muscle and nutrition index after radical cystectomy for patients with urothelial carcinoma of the bladder. BMC Cancer 2017;17:237.

5. Lee SM, Russell A, Hellawell G. Predictive value of pretreatment inflammation-based prognostic scores (neutrophil-to-lymphocyte ratio, platelet-to-lymphocyte ratio, and lymphocyte-to-monocyte ratio) for invasive bladder carcinoma. Korean J Urol 2015;56:749-55.

6. Smith AB, Deal AM, Yu H, et al. Sarcopenia as a predictor of complications and survival following radical cystectomy. J Urol 2014;191:1714-20.

7. Osland E, Hossain MB, Khan S, et al. Effect of timing of pharmaconutrition (immunonutrition) administration on outcomes of elective surgery for gastrointestinal malignancies: a systematic review and meta-analysis. JPEN J Parenter Enteral Nutr 2014;38:53-69.

Cite this article as: Miyake M, Fujimoto K. Pretreatment assessment and perioperative nutritional interventions for patients with bladder cancer undergoing radical cystectomy. Transl Androl Urol 2018;7(6):984-986. doi: 10.21037/ tau.2018.07.02
8. Zhang Y, Gu Y, Guo T, et al. Perioperative immunonutrition for gastrointestinal cancer: a systematic review of randomized controlled trials. Surg Oncol 2012;21:e87-95.

9. Munbauhal G, Drouin SJ, Mozer P, et al. Malnourishment in bladder cancer and the role of immunonutrition at the time of cystectomy: an overview for urologists. BJU Int 2014;114:177-84.

10. Roth B, Birkhauser FD, Zehnder P, et al. Parenteral nutrition does not improve postoperative recovery from radical cystectomy: results of a prospective randomised trial. Eur Urol 2013;63:475-82.

11. Maffezzini M, Gerbi G, Campodonico F, et al. Multimodal perioperative plan for radical cystectomy and intestinal urinary diversion. I. Effect on recovery of intestinal function and occurrence of complications. Urology 2007;69:1107-11.

12. Maffezzini M, Gerbi G, Campodonico F, et al. A multimodal perioperative plan for radical cystectomy and urinary intestinal diversion: effects, limits and complications of early artificial nutrition. J Urol 2006;176:945-8; discussion 948-9.

13. Marik PE, Flemmer M. The immune response to surgery and trauma: 344 Implications for treatment. J Trauma Acute Care Surg 2012;73:801-8.

14. Miura T, Mitsunaga S, Ikeda M, et al. Characterization of patients with advanced pancreatic cancer and high serum interleukin-6 levels. Pancreas 2015;44:756-63. 